\title{
The instructive extracellular matrix of the lung: basic composition and alterations in chronic lung disease
}

\author{
Gerald Burgstaller ${ }^{1}$, Bettina Oehrle ${ }^{1}$, Michael Gerckens ${ }^{1}$, Eric S. White ${ }^{2}{ }^{2}$, \\ Herbert B. Schiller ${ }^{1}$ and Oliver Eickelberg (1) ${ }^{3}$
}

Affiliations:

${ }^{1}$ Comprehensive Pneumology Center, University Hospital of the Ludwig-Maximilians-University Munich and Helmholtz Zentrum München, Member of the German Center for Lung Research, Munich, Germany.

${ }^{2}$ Division of Pulmonary and Critical Care Medicine, Department of Internal Medicine, University of Michigan Medical School, Ann Arbor, MI, USA.

${ }^{3}$ Division of Respiratory Sciences and Critical Care Medicine, University of Colorado, Denver, CO, USA.

\section{Correspondence:}

Gerald Burgstaller, Comprehensive Pneumology Center, Helmholtz Center Munich, Ludwig Maximilians University Munich, University Hospital Grosshadern, Max-Lebsche-Platz 31, Munich, Germany.

E-mail: gerald.burgstallerahelmholtz-muenchen.de

\section{@ERSpublications}

Molecular/biomechanical alterations within ECM in chronic lung diseases direct cellular function/ differentiation http://ow.ly/9GrY30c0LJG

Cite this article as: Burgstaller G, Oehrle B, Gerckens M, et al. The instructive extracellular matrix of the lung: basic composition and alterations in chronic lung disease. Eur Respir J 2017; 50: 1601805 [https://doi. org/10.1183/13993003.01805-2016].

ABSTRACT The pulmonary extracellular matrix (ECM) determines the tissue architecture of the lung, and provides mechanical stability and elastic recoil, which are essential for physiological lung function. Biochemical and biomechanical signals initiated by the ECM direct cellular function and differentiation, and thus play a decisive role in lung development, tissue remodelling processes and maintenance of adult homeostasis. Recent proteomic studies have demonstrated that at least 150 different ECM proteins, glycosaminoglycans and modifying enzymes are expressed in the lung, and these assemble into intricate composite biomaterials. These highly insoluble assemblies of interacting ECM proteins and their glycan modifications can act as a solid phase-binding interface for hundreds of secreted proteins, which creates an information-rich signalling template for cell function and differentiation. Dynamic changes within the ECM that occur upon injury or with ageing are associated with several chronic lung diseases. In this review, we summarise the available data about the structure and function of the pulmonary ECM, and highlight changes that occur in idiopathic pulmonary fibrosis (IPF), pulmonary arterial hypertension $(\mathrm{PAH})$, chronic obstructive pulmonary disease (COPD), asthma and lung cancer. We discuss potential mechanisms of ECM remodelling and modification, which we believe are relevant for future diagnosis and treatment of chronic lung disease. 


\section{Part A: the pulmonary ECM}

The evolution of complex tissues in higher organisms is accompanied by an expansion in protein diversity and structural organisation in the ECM [1], as well as an expansion of diversity of ECM receptors on cells. As in all other organ systems, the pulmonary ECM is organised into two main structural types: 1) basement membranes, which are thin sheets of glycoproteins that cover the basal side of epithelia and endothelia, and surround muscle, fat and peripheral nerve cells; and 2) interstitial matrices, which form a loose and fibril-like meshwork that interconnects structural cell types within tissues, and thereby maintains the three-dimensional (3D) cohesiveness and biomechanical characteristics of the lung [2]. Both basement membranes and interstitial matrices form tissue-specific "niches" that influence the stemness

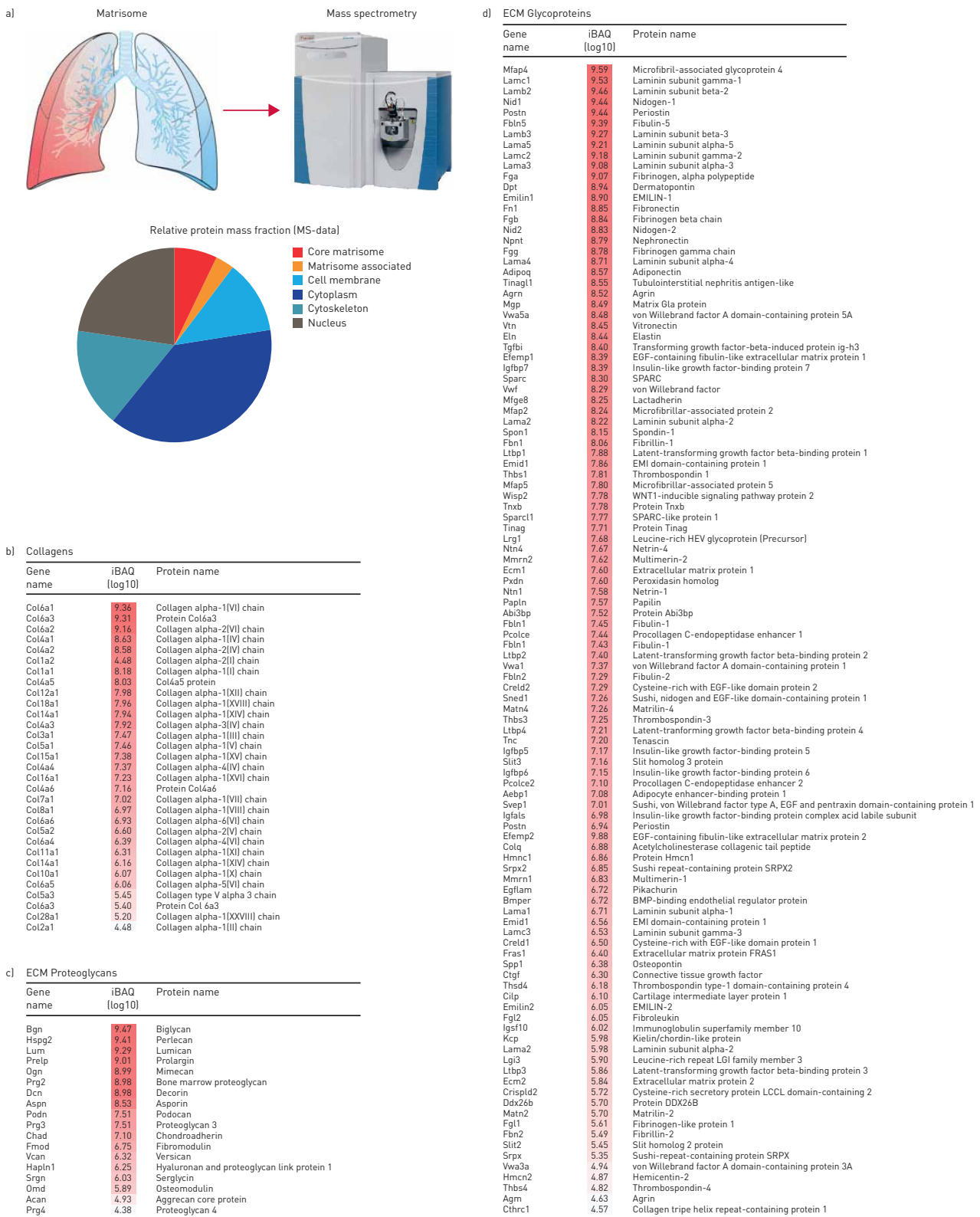

FIGURE 1 Extracellular matrix (ECM) proteome of the adult healthy murine lung. a) We used our recently published proteomic dataset of murine lung proteomes to calculate the relative protein mass fraction of the indicated gene categories. The MS intensities of the proteins within the depicted gene categories were expressed as parts per million fraction of the total MS intensity of all proteins. b-d) To determine the relative copy numbers of ECM proteins in the lung proteome, MS intensities were normalised to the theoretical number of tryptic peptides ( protein size normalisation; iBAQ). We used this protein copy number estimation to rank collagens (b), proteoglycans (c) and glycoproteins (d) by their abundance. MS: mass spectrometry; iBAQ: intensity-based absolute quantification. 
and differentiation of progenitor/stem cell populations, as well as the proper function of tissue/ compartment-specific differentiated cell types.

\section{Unravelling the matrisome}

Based on bioinformatic analysis of the available mammalian genome, the number of core structural components of the entire mammalian ECM is predicted to include 2300 proteins (core matrisome) $[3,4]$. In addition, the ECM associates with and serves as a reservoir for a large number of secreted proteins, such as growth factors, ECM-modifying enzymes or other ECM-associated proteins, which do not contribute to the structure of ECM but affect its function as an instructing "niche" (matrisome-associated proteins). Owing to their complex biochemical properties (insolubility, high molecular weight and high degree of protein cross-linking), a detailed analysis of ECM proteins used to be very challenging [5]; however recent progress in mass spectrometry (MS) equipment and quantification algorithms has, for the first time, enabled a comprehensive analysis of ECM proteomes from various tissues [6-13]. Label-free protein quantification by MS not only allows relative comparison of proteins across samples and experimental conditions, but also allows estimation of absolute amounts [14]. Using this approach, we analysed our recently published proteomic dataset of the mouse lung proteome [12] and ranked the relative abundance of core matrisome proteins, providing an in-depth ECM expression profile of healthy mouse lung (figure 1).

\section{Composition of the ECM}

Collagens constitute a major portion of the protein content of the lung [15], and are severely altered in many lung diseases (figure 2). The fibrillar collagens (types I, II, III, V and XI), which have great tensile strength but low elasticity, contribute to the overarching architecture of the lung $[16,17]$, whereas the large elastic fibres, which are characterised by low tensile strength and high elasticity, provide the lung with its necessary compliance and elastic recoil. Elastic fibres are composed of two distinct components: the ECM protein elastin is found in its crosslinked form in the inner core of elastic fibres, whereas the outer periphery of elastic fibres contains $10-15 \mathrm{~nm}$-sized microfibrils [18].

The major structural components of microfibrils are the large glycoproteins fibrillin-1, -2 and -3 [19]. Additionally, other proteins such as microfibril-associated glycoproteins, fibulins, elastin microfibril interface-located proteins (EMILINs) and members of the elastin-crosslinking lysyl oxidase (LOX) family are associated with microfibrils or with elastin itself [18]. Interestingly, microfibrillar-associated protein 4 (MFAP4) was the most abundant glycoprotein found in our matrisome dataset of healthy adult mouse lungs (figure 1) [12]. In humans, high expression of MFAP4 has been shown not only for lung, but also for other highly elastic tissues such as heart and intestine [20]. The biological function of MFAP4 is largely unknown; although it has been identified as a serum biomarker for haptic fibrosis, it fails as a biomarker for lung fibrosis [21].

The interstitial ECM of the alveoli is composed of a relaxed meshwork, largely based on type I and III collagens and elastin as important core proteins [22, 23]. The 3D arrangement of this intertwined fibre network allows nonlinear stress-strain behaviours (hysteresis and viscoelasticity) that are a characteristic property of soft connective tissues [24]. During respiration, the energy dissipation that causes hysteresis and viscoelasticity in the lung parenchyma is driven by fibre-fibre contacts in the collagen-elastin ECM, although contractile cell types and surfactant in the air-liquid interface may also contribute to these effects [17]. In addition to their biomechanical functions, elastic fibre components, such as fibulins and EMILINs, govern cell-ECM adhesion by interacting with heteromeric transmembrane integrin receptors [25, 26]. In contrast to EMILINs, which bind integrins via their gC1q-1 domain, fibulin-5 binds integrins via an evolutionally conserved RGD (arginine-glycine-aspartic acid) sequence. This RGD tripeptide-binding motif is characteristic of other ECM glycoproteins, including fibronectin, vitronectin, osteopontin, collagens, thrombospondins, fibrinogen and von Willebrand factor, all of which mediate cell-ECM adhesion via integrin receptors [27]. Apart from fibrous collagens and glycoproteins, proteoglycans (PGs), which consist of a core protein component covalently linked to sulfated polysaccharides or glycosaminoglycans (GAGs), are major constituents of the ECM [28]. Owing to their high polysaccharide content, PGs are hydrophilic, thereby enabling hydrogel formation and contributing to the viscoelasticity of the lung. PGs contain the basement membrane constituents perlecan and agrin, as well as the hyalectans versican, aggrecan, neurocan, brevican and the subclass of small-leucine rich proteoglycans, including its most prominent members, decorin, biglycan and lumican [29]. Decorin, biglycan and lumican were among the most abundant proteoglycans in our matrisome dataset of healthy adult mouse lung (figure 1) [12]. In vitro, both human decorin and biglycan are able to bind to the profibrotic cytokine transforming growth factor- $\beta 1$ (TGF- $\beta 1$ ), but in vivo, only decorin but not biglycan was shown to inhibit the fibrogenic effect of TGF- $\beta 1$ [30]. Furthermore, decorin reportedly interacts with various 

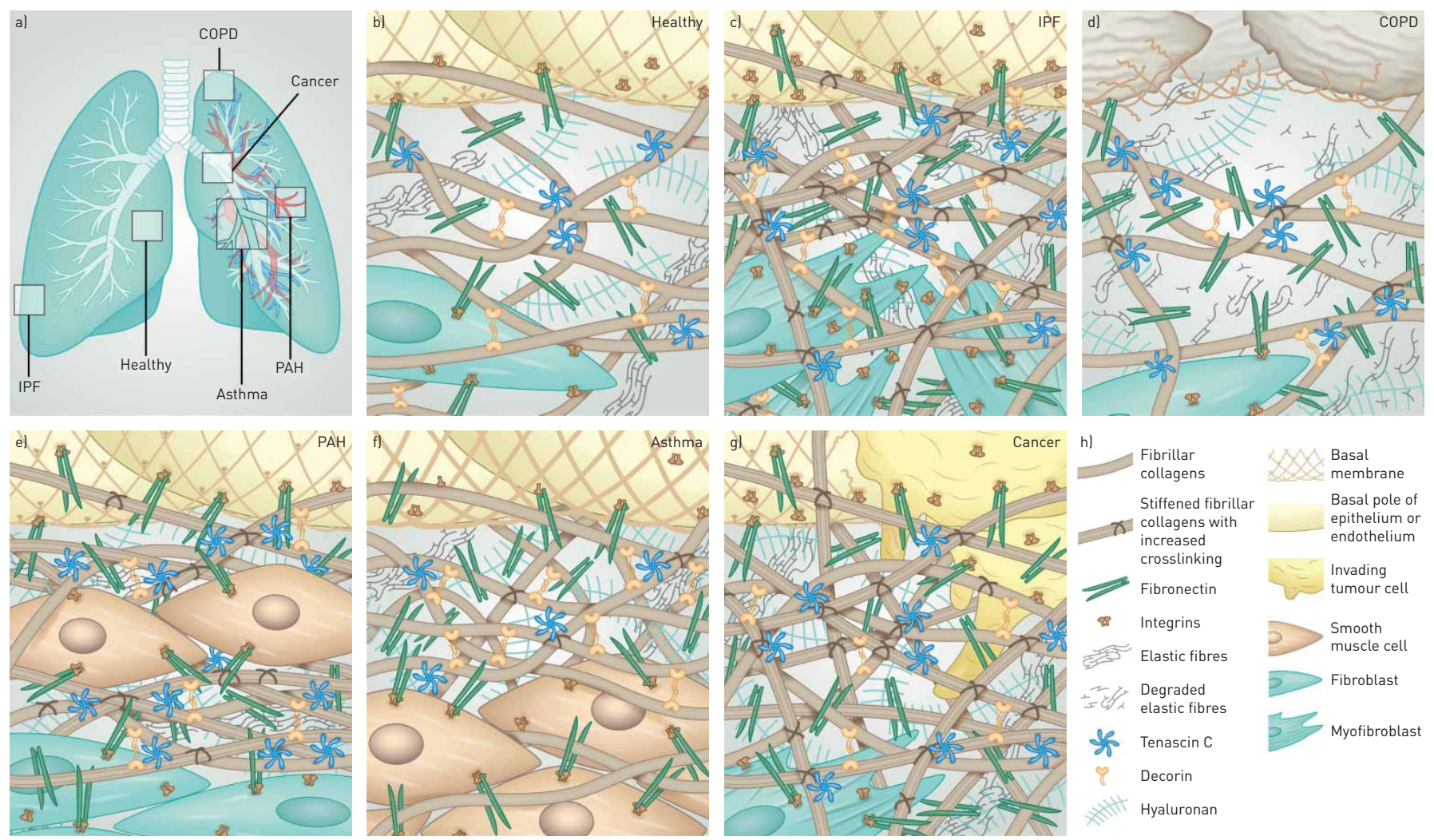

FIGURE 2 Pathological changes within the interstitial extracellular matrix (ECM) in the diseased lung. a) Overview of the lung and sites of disease formation. b) Healthy interstitial ECM is maintained by the activity of resident fibroblasts and represents a loose meshwork of collagens, elastin and fibronectin anchored to the basal membrane of the epithelial cell layer. c) In idiopathic pulmonary fibrosis (IPF), fibroblasts transdifferentiate to highly contractile myofibroblasts, which deposit high levels of ECM molecules into the interstitium and dramatically increase ECM rigidity by enzymatic covalent crosslinking of collagen and elastin. d) The hallmarks of pathological changes within the ECM in chronic obstructive pulmonary disease (COPD) are the extensive destruction of elastic fibres by ECM-degrading enzymes released by inflammatory cells, along with elevated levels of hyaluronan and tenascin C, and decreased deposition of decorin. The destruction of the epithelial cell layer leads to air space enlargements (emphysema). e) In pulmonary arterial hypertension (PAH), remodelling of the ECM within the arterial wall is characterised by an increase in elastin and collagen fibres, fibronectin, and tenascin C, and hyperplasia of smooth muscle cells. f) In asthma, the characteristic ECM changes take place beneath the bronchial epithelium and thickened basal membrane. In addition, smooth muscle cells undergo hyperplasia, and deposition of collagens, fibronectin, hyaluronan and decorin are increased. g) In cancer, tumours at primary and metastatic sides are surrounded by an extensive stiff stroma that contains highly crosslinked collagens, and high levels of fibronectin, tenascin C and hyaluronan. h) Legend depicting molecules and cell types.

metalloproteases and can act as a tumour suppressor by attenuating tumour growth, migration and angiogenesis [31].

\section{Post-translational modifications of ECM components}

Post-translational modifications (PTMs), e.g. enzymatic and chemical crosslinking, transglutamination, glycation and glycosylation, oxidation, and citrullination, are known to affect the structural and/or functional diversity of ECM proteins [32]. For instance, enzymatic cross-linking of collagen and elastin, a substantial step during their biosynthesis, which is mainly exerted by the enzymes of the lysyl oxidase family (LOX, LOXL1-4) and the transglutaminase family members (TG1-7, FXIII-A), provides ECM components with their characteristic tensile strength [33-35]. Furthermore, the enzymatic (glycosylation) or non-enzymatic (glycation) addition of sugars to proteins also impacts on the biomechanics and function of the ECM. In particular, glycation is currently recognised as a factor in ageing-related fibrosis. This process causes the formation and accumulation of advanced glycation end-products (AGEs). Aberrant glycation processes have been associated with increased tissue stiffness, e.g. in experimental AGE formation in tendons, which were shown to reduce fibre sliding while increasing fibre stretching [36]. Carbamylation, which is a non-enzymatic addition of urea to isocyanic acid residues of proteins, negatively influences the stability and conformation of collagen I triple helices, as well as their degradation by MMPs [37]. The oxidation of ECM proteins by reactive oxygen species (ROS) further modulates qualities of the ECM by altering its production, turnover and modifications, as well as having an impact on cell-ECM interactions [38]. Additionally, serum levels of oxidative stress markers have been found to be elevated in 
patients with IPF [39] and in the breath condensates of patients with asthma [40]. The ECM can also be modulated by citrullination, an enzymatic deamination reaction of arginine that affects cell adhesion [41]. For a more comprehensive overview of PTMs in the ECM and their roles in disease, we refer the reader to these additional reviews [32, 42-45]. Taken together, the structural and functional properties of the pulmonary ECM can be modified by PTMs, which are considered an important potential contributor to lung disease, as outlined in detail in the following sections.

\section{Biomechanics and cell-ECM crosstalk}

All cells are attached transiently or permanently to some form of ECM via cell adhesion receptors, most prominently the integrin family of transmembrane heterodimeric receptors [46, 47]. Cells can use integrins to probe both the biochemical and topographical characteristics of their environment [47,48]. Signal transduction emanating from cell-ECM adhesions controls cellular behaviour, such as motility and spreading, morphology, survival, proliferation and differentiation [1,22]. These signalling events depend on a multitude of proteins that are recruited to plasma membrane integrin clusters, which are collectively termed the "adhesome" [49]. Recent proteomic studies have delineated the overall protein composition of the adhesome and its dynamics under the influence of mechanical forces [50-55]. When cells encounter their extracellular substrate, they react to the stiffness of that substrate in multiple ways, including immediate cell shape and activity changes, as well as long-term gene expression and cell-identity changes. This mechano-reciprocity is generated by feedback connections between cell-ECM adhesions and the cytoskeleton, which tune the strength of contractile forces to an equilibrium between the applied force and the tensile strength of the ECM substrate [47]. In this process, the structure and organisation of the cytoskeleton is altered, and this has been shown to induce long-term gene expression changes [56]. The precise molecular nature of many elements in these feedback connections is currently unknown, and it is also unclear to what extent the equilibrium of mechano-sensing to mechano-response differs between cell types and how such differences can be programmed. Importantly, it is well appreciated that mechano-sensitive signalling pathways are important in the pathophysiology of fibrotic diseases and cancer, both of which exhibit dynamic changes in ECM composition and mechanical properties during disease progression [57-61]. In particular, pathologically elevated ECM deposition, accompanied by covalent cross-linking and ECM remodelling, generates a dramatic increase in ECM stiffness, thus causing a mechanical gradient between pathological and normal tissue. Stiffness gradients within the ECM dictate the migratory behaviour of cells in a process called durotaxis. Durotaxis seems to be a rather general phenomenon, as many cell types, including fibroblasts, mesenchymal stem cells (MSCs), myoblasts and cancer cells, have been shown to migrate along stiffness gradients [62-65]. Furthermore, ECM stiffness influences fibroblast spreading, contractility and differentiation $[66,67]$. When grown on soft matrices, fibroblasts and many other cells usually exhibit low contractile forces together with reduced spreading and proliferation $[68,69]$. ECM rigidity also affects differentiation of MSCs, as osteogenic and adipogenic lineages appear on rigid and soft surfaces, respectively [66]. Primary cultures of alveolar epithelial type II (ATII) cells, which are thought to possess stem-cell potential and are capable of long-term self-renewal in the adult lung, alter their morphology and functional characteristics (surfactant synthesis and secretion) dependent on the ECM substrate used in culture [28]. In an in vitro study, primary human alveolar macrophages, which mediate lung fibrosis, showed increases in phagocytosis and changes in transcription and phenotype when cultured on rigid ECM [70]. Furthermore, for a monolayer of endothelial cells, it was demonstrated that their cultivation on stiffer ECM had negative effects on the monolayer integrity, thus advocating for a significance of barrier permeability in respect to transmigration of leukocytes [71].

\section{The reservoir function of the ECM}

The ECM serves as a reservoir for a number of growth factors and cytokines, which are crucial for cell differentiation and proliferation $[4,22,72]$. The association of factors such as fibroblast growth factor (FGF), hepatocyte growth factor, and latent and active TGF- $\beta 1$ (for a complete list we refer the reader to a previously published table [72]) with the ECM poses some consequences: ECM-bound factors 1) can be latent, masked or have a different activity compared with their soluble form, 2) can form immobilised gradients crucial for cell migration, 3) might, as imprints of former cellular activity, have a memory function for instructing cellular behaviour, 4) facilitate cell adhesion and cell growth, and 5) as stored factors, may be activated rapidly by proteolytic release and generate local signals that are independent of slow processes such as gene expression [72, 73]. Degradation of the ECM is a pivotal event in tissue remodelling, and in this process the zinc-dependent proteinases (metzincins) play a decisive role.

\section{ECM-degrading enzymes}

This superfamily of metalloproteinases comprises the matrix metalloproteinases (MMPs) and adamalysins. MMPs are a family of 25 zinc-dependent endopeptidases. These enzymes are able to cleave all ECM components [74] and basement membranes [75]. MMPs are secreted in a highly concerted process along 
with their inhibitors, the tissue inhibitors of metalloproteinases (TIMPs) [76]. Adamalysins comprise the disintegrin and metalloproteinases (ADAMs) and ADAMs with a thrombospondin motif (ADAMTS). Owing to the capability of ADAMs to shred cytokines, growth factors and FAS ligand, a potential clinical application has been ascribed to them in fibrosis, cancer, inflammation and neurodegeneration [77]. In fibrotic lung diseases, the production and secretion of MMPs and TIMPs has been attributed to macrophages [78], fibrocytes, endothelial cells and fibroblasts [79], and strong expression of MMPs, especially MMP-1 and MMP-7, has also been observed in bronchiolar and alveolar epithelial cells [80]. For a more detailed discussion about molecular players in ECM degradation and remodelling, the reader is referred to the excellent review by Lu et al. [81].

\section{Part B: ECM remodelling processes in chronic lung diseases IPF \\ Pathogenesis and tissue remodelling}

While the aetiology of IPF remains largely unknown, IPF affects roughly five million people worldwide. Until recently, lung transplantation was the only effective therapy. Recently, two Food and Drug Administration (FDA)-approved drugs, pirfenidone and nintedanib, which have pleiotropic effects on cells including inhibition of ECM production, have been shown to slow the rate of decline in lung function [82, 83]. In IPF, repetitive lung injury and subsequent repair mechanisms lead to an ongoing destruction of elastic parenchymal lung tissue, which is constantly replaced by stiff scar tissue, comprising large and condensed cell-ECM aggregates known as fibroblastic foci within the pulmonary interstitium (figure 2c). Progressive tissue remodelling leads to traction bronchiectasis, thickened interlobular septae and subpleural honeycombing, which can be imaged by computed axial tomography (CAT) [84]. All these microscopic and macroscopic changes contribute to a progressive loss in gas exchange, with subsequent deteriorating lung function, breathlessness and finally respiratory failure leading to death [85].

\section{Myofibroblasts}

Fibroblastic foci contain activated fibroblasts and/or highly contractile myofibroblasts, which are mainly characterised by their neo-expression of $\alpha$-smooth muscle actin ( $\alpha$-SMA) and its incorporation into contractile stress fibres. Apart from their contractile features, myofibroblasts produce high amounts of ECM molecules in response to pro-fibrotic stimuli that are thought to result from imbalanced repair mechanisms of the alveolar and bronchiolar epithelia following repeated microinjuries and inflammatory processes [86] (figure 2c). The origin of myofibroblasts in fibrotic foci is still under heavy debate. In various studies, myofibroblasts in the fibrotic lung have been shown to stem from bone marrow-derived progenitor cells [87], peripheral blood circulating fibrocytes [88, 89], alveolar epithelial cells undergoing epithelial-mesenchymal transition [90], resident fibroblasts [91] and pericytes [92]. Quite controversially, by applying lineage tracing, a different study described an as yet unidentified population of resident stromal cells within fibrotic foci as the source of myofibroblasts, but ruled out pericytes and epithelial cells as sources [93].

\section{Instructiveness}

As the myofibroblast is considered a hallmark of IPF, it is conceivable that the ECM produced by the myofibroblast is an instructive disease component, which perpetuates disease progression. Indeed, by culturing fibroblasts originating from either IPF or healthy control lungs on either fibrotic or healthy decellularised lung tissue, PARKER et al. demonstrated that the aberrant ECM microenvironment in IPF predominantly drives changes in gene and protein expression, rather than intrinsic cellular alterations [94]. The process of decellularisation and successful recellularisation of whole organs, ideally with autologous stem or progenitor cells, was tested as a potential surrogate in lung transplantation medicine [95-97]. Even cadaveric human lungs, which were taken from individuals with chronic lung diseases, have been investigated for possible usage in ex vivo tissue engineering [98]. However, in light of current findings that decellularised ECM scaffolds from patients with IPF alter the transcriptome and translatome of reseeded fibroblasts, it remains an intriguing open question as to whether such surrogates derived from pathological lung tissues might be usable for lung transplantation at all.

\section{Mechanophysiology}

The current concepts in the field assume crucial functions of specific ECM proteins in fine-tuning signalling pathways by their capacity to modulate growth factor signalling [99-101], or important effects of tissue stiffening on disease progression. ECM rigidity in fibrotic disorders is increased by enzymatic covalent crosslinking of collagen and elastin by LOX and TG family members $[34,35]$. In IPF, expression of lysyl oxidase-like 2 (LOXL2) and TG2 has been shown to be increased in the serum and lungs, respectively, of patients with IPF $[102,103]$. The stiffness of decellularised IPF tissue is indeed higher than normal, as shown by atomic force microscopy [13], and correspondingly, the activity of non-muscle 
myosin-II, which drives cell contractility, was shown to be also higher in fibrotic areas of patients with IPF [57]. Thus, the activity and survival of activated fibroblasts in fibrotic diseases may be largely controlled by mechanical signals. Indeed, inhibition of myosin-II-mediated cell contractility using a Rho-kinase inhibitor induced myofibroblast apoptosis and ameliorated experimental lung fibrosis [57]. TSCHUMPERLIN and colleagues used atomic force microscopy measurements on experimental fibrosis in bleomycin-injured lungs to reveal a striking increase in fibrotic zones with an elastic modulus (which measures tissue stiffness in Pascals) exceeding $2 \mathrm{kPa}$ [68]. Interestingly, a physiological stiffness range (0.2-2 kPa) keeps lung fibroblasts in a quiescent state, whereas substrates with higher stiffness $(2-35 \mathrm{kPa})$, as observed in fibrotic lungs, induce a pro-fibrogenic phenotype with high proliferation and ECM synthesis rates [68]. We have shown that (myo)fibroblasts require a combination of $\alpha 5 \beta 1$-integrin and $\alpha \mathrm{V}$-integrin family heterodimers to effectively respond to the stiffness of a fibronectin-rich tissue microenvironment as is present in tissue fibrogenesis [50]. Interestingly, ablation of $\alpha \mathrm{V}$-family integrins reduced fibrosis in several organs, including the lung, in experimental injury mouse models [104], which might indicated that these integrins function in the mechano-sensing response [50] and/or TGF- $\beta$ activation [105].

\section{Proteomic ECM signatures}

Future proteomic investigations of acute versus chronic models of lung injury and the ensuing fibrosis might enable the identification of extracellular niche components that selectively drive pathology in a permanent and progressive scarring phenotype, as seen in IPF. We performed a time-resolved proteome study of the acute bleomycin lung injury mouse model and, based on the observed temporal kinetics of protein abundance, we were able to predict functions of individual proteins in the early stages of tissue repair, such as stem cell mobilisation, or late stages, such as resolution of fibrosis. Unexpected ECM proteins that were not studied in the context of injury versus repair, and fibrosis, such as Emilin-2 and collagen-XXVIII, were discovered to be highly upregulated 2 weeks after injury [12]. By using a combination of stable isotope labelling, solubility fractionation of decellularised lung tissue and proteomic analysis, DECARIS and colleagues assessed the kinetics of ECM synthesis and deposition in a bleomycin model [106], and found that the protein synthesis rates of fibrillar collagens (types I, III and V) were dramatically increased in both the soluble and insoluble fractions during the late fibrotic response, whereas the small leucine-rich proteoglycans biglycan and decorin, as well as fibronectin, were elevated in the early fibrotic response. However, in a different MS-based ECM analysis of decellularised IPF lungs, the authors identified an enrichment of glycosaminoclycans, matrix-Gla protein and microfibrillar-associated proteins in the ECM of IPF lungs [13]. Conclusively, the ECM of diseased human lungs may harbour a diseaseand progression-specific combination of secreted proteins and proteolytic fragments of ECM components ("signature"), which may not only be of great diagnostic and/or prognostic value for future precision medicine but might also give novel insights into molecular mechanisms in disease pathogenesis.

\section{COPD and emphysema}

The pathophysiological aspects of COPD, which is mainly caused by smoke exposure, include airflow obstruction and hyperinflation. The airflow limitation in COPD is caused by three inter-related processes: small airway remodelling leading to a thickening of airway walls, the loss of small airways and the enlargement of the respiratory air spaces in the alveoli, a condition called emphysema [17]. Consequently, the transpulmonary pressure dissemination is disrupted, causing hyperinflation of the lungs. These structural alterations cause biomechanical changes that manifest as loss of the elastic recoil of the lung. As ECM represents the main stress-bearing component in the respiratory system, the loss of elastic recoil in pulmonary emphysema suggests that elastin, the core component within elastic fibres, is the major target of decomposition (figure 2d). In a mouse disease model of emphysema, both intra-tracheal instillation of the elastolytic enzyme elastase and a deficiency of $\alpha 1$-antitrypsin, the main inhibitor of neutrophil elastase, resulted in the formation of emphysema [107]. The imbalance of protease and antiprotease activity is a widely accepted hypothesis of how tissue decomposition might occur in emphysema [108]. The release of ECM-degrading enzymes is largely attributed to inflammatory cells, mainly macrophages, neutrophils and $\mathrm{T}$ cells [109]. However, it may not be only the destruction of elastic fibres that is responsible for the changes in lung biomechanics, as there is also experimental evidence that collagen remodelling within the alveolar walls contributes to the structural and biomechanical changes found in pulmonary emphysema [110]. Accordingly, a thickening of collagen fibrils has been demonstrated on an ultrastructural level in humans and in rodent mouse models [111]. This quite surprising finding is supported by in vivo evidence that elastase degradation of ECM components can trigger collagen synthesis [112], but the described response might also be a result of TGF- $\beta 1$-producing macrophages that stimulate the collagen production of effector cells [111]. However, different compartments of the lungs are differently affected by aberrant collagen deposition in COPD. Whereas electron micrographs revealed a high abundance of collagen and elastin in alveolar septal walls of emphysematous tissue [113], lower expression of collagen I was found in airways of patients with COPD [114]. ANnoni et al. analysed the detailed composition of the ECM from patients with 
COPD compared with healthy controls in various lung compartments, and showed that in samples of moderate COPD, there were alterations in elastic fibres, fibronectin, collagens, tenascin-C and versican throughout all lung compartments [114]. In accordance with these findings, Roman et al. demonstrated nicotine-induced upregulation of fibronectin expression both in vitro and in vivo [115]. Moreover, in vitro, airway smooth muscle cells (ASMs) isolated from patients with COPD produced higher levels of Col8a1, MMP1, MMP3 and MMP10 in response to cigarette smoke extract [116]. Recently, mice with a deficiency of the ECM glycoprotein fibulin-4 were found to have increased MMP and neutrophil elastase activity in the lungs, which led to alveolar breakdown in newborn mice and subsequently to the formation of emphysema [117]. In COPD, expression levels of osteopontin, which is a phosphorylated acidic ECM glycoprotein with functions in cell adhesion and migration, was found to be elevated in the sputum of patients with COPD [118]. Increased levels of osteopontin are not specific for COPD, as similar upregulation of osteopontin was described in the lungs and bronchoalveolar lavage fluid of patients with IPF [119]. AGEs, which are mostly produced by inflammatory processes, were found to accumulate in the skin of patients with COPD. This potentially implies a role of AGEs and glycation events in the early pathological phases of COPD, although direct implication of AGEs in the ECM in vivo and in human lung still remains unclear [120]. Furthermore, during acute exacerbations of COPD, an accelerated turnover of ECM protein occurs, leading to protease-generated fragmentation of ECM proteins such as collagens, versican and elastins. These blood-circulating ECM protein fingerprints might be utilised as potent biomarkers in predicting COPD disease progression [121].

\section{PAH}

$\mathrm{PAH}$ is a progressive disease with a poor prognosis. The pathophysiology of PAH is characterised by an increased workload in the right heart ventricle as a result of several processes, such as genetic predisposition, inflammation, cell proliferation, vasoconstriction and vascular remodelling. The pulmonary arteries are affected by persistent vasoconstriction and vascular remodelling, resulting in reduced pulmonary arterial compliance [122]. In $\mathrm{PAH}$, the whole three-layered architecture of the arterial wall undergoes remodelling, thus leading to intimal, medial and adventitial thickening [123] (figure 2a). Accordingly, dynamic incorporation studies using radioactive tracer revealed rapid synthesis of elastin and collagen in pulmonary arteries during hypoxic pulmonary hypertension [124]. Furthermore, the ECM component tenascin- $\mathrm{C}$ has been associated with disease progression. In patients with $\mathrm{PAH}$ and in experimental disease models alike, tenascin-C correlated with smooth muscle cell (SMC) proliferation (figure 2e) and mitogenic response to FGF2. Likewise, in fibrotic mouse lungs tenascin-C negatively correlated with lung compliance, that is, larger amounts of this protein were associated with stiffer lung tissue [12]. Meanwhile, a positive correlation of tenascin-C expression and SMC proliferation was found in patients with congenital heart disease and in the monocrotaline-induced PAH rodent model [125]. However, in vitro elastin expression inversely correlated with the proliferation of SMCs [126]. Additionally, within the thickened intima of affected vessels from lung biopsy tissues from patients with $\mathrm{PAH}$, elevated levels of fibronectin and tenascin-C were identified by immunohistochemistry and in situ hybridisation [125]. Increased deposition of GAGs such as hyaluronic acid (HA) has also been observed in remodelled pulmonary arteries $[127,128]$. Furthermore, a pathological heavy chain modification of HA, which has also been suggested to promote leukocyte adhesion and activation, was identified in the lung tissue of patients with PAH [129].

It is known that MMPs play a decisive role in pulmonary vascular remodelling. In vitro and in situ studies on pulmonary arterial SMCs revealed an imbalance of MMPs and TIMPs in idiopathic PAH [130]; MMP3 was found to be down-regulated, whereas TIMP1 was up-regulated in the affected SMCs [131]. NAVE et al. reported that lysyl oxidases are also involved in pulmonary vascular remodelling in $\mathrm{PH}$, inducing massive cross-linking of the ECM [132]. Despite the vascular wall hyperplasia and hypertrophy found in pulmonary hypertensive lungs, these have been successfully decellularised and recellularised with mesenchymal stem cells [133]. When successfully recellularised with stem cells, decellularised 3D ECM scaffolds from diseased lungs might pose a valid source in clinical use to generate healthy functional tissues. However, it is a prerequisite that the recellularisation process leads to successful remodelling of the diseased hypertrophic ECM in the decellularised scaffolds, and at least in fibrotic diseases, the contrary effect was observed [94].

\section{Asthma}

The pathology of asthma is characterised by chronic inflammation of submucosal regions of the small airways of the lung, with associated goblet cell hyperplasia, augmented mucus secretion, smooth muscle hypertrophy and bronchoconstriction (figure 2f). The lung structure is affected by airway remodelling caused by ASM hyperplasia, with a concomitant modified ECM profile around the airway structures. These structural alterations manifest in biomechanical dysfunctions. Accordingly, the elastic recoil of the 
lungs is diminished, which might be caused by impairment of the elastic load transmission between the lung parenchyma and airways. Aberrant accumulation of ECM was previously found in asthmatic lungs, with its main localisation to the submucosal and adventitial areas of both large and small airways [134]. ECM deposits, which occur even in mild asthma, include collagens I, III and V in the subepithelial region, as well as fibronectin in the lamina reticularis of the bronchial epithelium [135] (figure 2f). Additionally, in fatal asthma, an increase in elastic fibres, MMP-9 and MMP-12 in the large airways was reported [136]. Interestingly, the ECM composition of the central and distal airways of patients with persistent asthma symptoms despite corticosteroid therapy (uncontrolled asthma) differs from that of patients with corticosteroid-susceptible (controlled) asthma. In essence, in uncontrolled compared with controlled asthma, deposition of collagen was found to be increased in the alveolar parenchyma, while versican was increased in the central airways and decorin and biglycan were increased in both compartments [137]. The differences between distal airway and bronchial remodelling might be based on the altered ECM secretion status of fibroblasts derived from those different areas [138]. These findings indicate a high clinical significance of ECM remodelling in asthma. It has been shown that in ASMs the presence of the soluble forms of fibronectin and collagen I can decrease the contractile but increase the proliferative capacity of ASMs, whereas culturing the cells in the presence of exogenous soluble laminin alone did not affect either proliferation or contractility [139]. However, a mechanism explaining how ECM proteins in their soluble, non-deposited forms differentially affect phenotype and contractile functions in ASMs remains elusive. Thus, changes in the ECM may drive mechanisms leading to an increased growth of ASMs (figure 2f), which is a pivotal event in the molecular pathology of asthma [140]. Furthermore, in vitro studies on ASMs suggest that alterations in the ECM also occur on post-translational levels in asthma [140]. The aberrant deposition of ECM components affects the biomechanical properties of the lung in different ways. ECM deposition around airways exaggerates the narrowing of the airways and induces alterations in tissue stiffness.

\section{Lung cancer}

The hallmarks of human cancers, which were comprehensively described by HANAHAN and WEINBERG [141], are multistep biological capabilities, which include sustained proliferation, evasion of growth suppression, death resistance, replicative immortality, induced angiogenesis, initiation of invasion and metastasis, and dysregulation of cellular energetics and inflammatory processes (figure 2g). As the ECM, by its biochemical and biophysical properties, is a regulator of the cellular responses that underlie cancer hallmarks, studying the influence of the ECM on neoplastic progression becomes essential [142]. For instance, small cell lung cancers (SCLCs) are surrounded by an extensive stroma of ECM, which harbours high levels of fibronectin, laminin, collagen IV and tenascin-C [143]. Furthermore, the tumour stroma of advanced sclerotic tumour tissue is rich in collagen I, whereas collagen III is highly abundant in less mature stroma [144]. Translational studies have identified that in patients with SCLC tumours, which are surrounded by an extensive stroma, the survival time was shortened, as binding of the SCLC cells to the ECM protects the cancer cells from chemotherapy-induced apoptosis [143]. Remodelling of tumour stroma strongly affects tissue stiffness, which in turn affects the mechano-biology of the cells and triggers cellular responses (figure $2 \mathrm{~g}$ ). As collagen is reported to be the main contributor to tensile strength within the lung architecture, collagen metabolism plays a decisive role in stiffening of the tumour stroma. Besides aberrant collagen deposition and turnover, alterations in collagen cross-linking have been observed in lung cancer. Most recently, CHeN et al. reported that tumour stroma exhibits high levels of hydroxylysine aldehyde-derived collagen cross-links (HLCCs) and lower levels of lysine aldehyde-derived cross-links (LCCs) [145] (figure 2g). Mechanistically, this shift from predominantly LCCs in healthy to HLCCs in tumorous lung tissue was traced back to the enzyme lysyl hydroxylase 2 (LH2). The concomitant increase in stroma stiffness in turn favoured tumour cell invasion and metastasis [145]. In line with these findings, tumour cells are known to express high LOX levels, and pharmacological interventions targeting this enzyme family repressed metastasis in tumour-bearing animals [146]. Mouw et al. showed that in breast cancer, ECM rigidity induced miR-18a to reduce expression of phosphatase and tensin homologue (PTEN), which in turn drove tumour progression [147]. Additionally, the increased ECM stiffness in tumours feeds back to cells in a Rho-dependent manner, increasing the cytoskeletal tension and stabilisation of focal adhesions [148]. As the tumour microenvironment is created by cancer and stromal cells, activation of the stromal cells occurs in an autocrine and paracrine fashion. In particular, activated fibroblasts, the so-called cancer-associated fibroblasts, play an essential role in tumour progression by substantially remodelling the tumour ECM, suppressing the immune response and releasing tumour growth-promoting factors [149]. Thus, the tumour ECM provides aberrant microenvironmental cues favouring proliferation and metastasis as well as inhibiting apoptosis of tumour cells. Accordingly, at primary and metastatic sites, the encapsulating tumour stroma can confer resistance to chemotherapy. It has been demonstrated in SCLC that the adhesion of tumour cells via integrin- $\beta 1$ to ECM proteins is a crucial event in cell resistance to chemotherapy [143]. Conclusively, the pathological changes in the ECM 
of lung cancers, such as increased collagen expression, altered collagen cross-linking and subsequent increase in tissue stiffness, are reminiscent of alterations also found in IPF, which advocates for similarities between these two diseases. Interestingly, many pathogenic analogies between cancer and IPF are reported: common risk factors (e.g. smoking, environmental exposure, viral infections and chronic tissue injury), delayed apoptosis, activation of specific signalling pathways, both epigenetic and genetic changes, altered expression of microRNAs, and aberrant cell proliferation and invasion [150-154]. There is a practical reasoning to consider IPF a cancer-like disease, which ultimately might lead to new clinical trials with cancer-related drugs for IPF [154].

\section{ECM model systems}

The composition of ECM and its biomechanical properties have an impact on cellular morphology [155], motility, spreading, cell viability and apoptosis, as well as proliferation and differentiation [5]. Therefore, as cellular behaviour and the ECM are highly interlinked, in vitro cell culture systems that exactly mimic the physiological and pathological conditions found in vivo are needed. Multiple in vivo animal models that reflect the pathogenesis of human lung diseases have been developed in the past. As a detailed discussion about animal models representing lung disorders would far exceed the scope of this review, we therefore refer the reader to a number of excellent reviews for further reading [156-162]. In particular, studies on mesenchymal cells, which are physiologically embedded within the ECM, may profit from in vitro, in vivo or ex vivo models that closely resemble the structure and biomechanics of pulmonary interstitial ECM [163-165]. A variety of these 3D cell culture systems has been applied in the past [166]. The most simplified model is tissue culture plastic coated with ECM molecules. Owing to its simplified design it can be used to study the interaction of specific ECM molecules with cells, although here the cells are attached to a two-dimensional (2D) surface rather than being embedded within an ECM. Moreover, the stiffness of cell culture plastic is far beyond that physiologically found in vivo; as measured by atomic force microscopy, Young's modulus (measured in Pascals) of cell culture plastic ranges from 2 to $4 \mathrm{GPa}$, whereas normal lung tissue varies between 0.44 and $7.5 \mathrm{kPa}$, depending on the region measured, and fibrotic human lung tissue from patients with IPF measures on average $16.52 \mathrm{kPa}[13,167,168]$. However, mechanically tunable 2D models using synthetic acrylamide- or polyethylene glycol-based matrices yield the ability to induce gradient formation and tight control of the ECM stiffness [166, 169]. Besides these artificial synthesised macromolecular structures, native and engineered biopolymers, consisting of collagens [170], elastin [171], fibrin [172] or laminin [173], are widely used for in vitro ECM model systems [174]. Basement membrane extract gels and decellularised fibroblast-derived matrices are also used nowadays [175], and these more closely mimic the composition, 3D structure and biomechanics of physiological ECM. However, none of these models can imitate the unique spatial geometry of pulmonary tissue. In order to circumvent these biomimetic limitations, vibratome-sliced ex vivo matrices taken from various animal disease models as well as from diseased human tissues can be used in their native or decellularised forms [13, 176-178]. Ex vivo models using decellularised lung tissue will help to further mechanistically delineate the impact of the ECM on lung pathologies. Finally, in the future, 3D bioprinting technologies or additive manufacturing techniques might hold promising and exciting new ways of creating biocompatible ECM scaffolds that biomechanically resemble real tissue. Such fabricated scaffolds could either be used as tunable in vitro models to study the instructive nature of the ECM, or ultimately, when successfully reseeded with cells, as a bioengineered tissue ready to replace diseased tissues or whole organs [179].

\section{Conclusion and future perspectives}

Chronic respiratory diseases originate from the complex interplay between environmental risk factors, epigenetic influences and genetic influences. Adverse environmental insults during foetal and infant life are thought to lead to enduring changes in lung structure and function. Such early life "programming" might aberrantly remodel the ECM and consequently pave the way for the development of chronic respiratory diseases in adulthood. The regulated remodelling of the ECM, especially in the process of wound healing, by its resident cells, is a prerequisite for maintenance of tissue homeostasis. In pathological events, qualities of the ECM, such as its composition and topological features, biomechanical properties, PTMs or role as a reservoir for secreted mediators, act as instructive cues that affect the behaviour of fibroblasts and other cell types. Once tissue homeostasis is disturbed by repeated injury and repair, aberrant feedback mechanisms between cells and their remodelled ECM trigger a vicious cycle that leads to disease progression. During disease progression, the interplay between cells and the ECM can be very complex, might involve various different cell types and can take place in spatially distinct compartments. For example, successful metastasis requires early remodelling of the ECM in the metastatic niche, which is spatially distant from the local niche of the primary tumour, in order to assist circulating tumour cells to engraft in distant organs [180]. Therefore, unravelling the molecular composition of the ECM in diseased lungs by means of state-of-the-art technologies, such as MS-driven proteomics or next-generation sequencing, is an important step towards the identification of diagnostic biomarkers for disease 
progression and therapy alike. Future attempts to develop methods for systems-level insights into the complex functional and physical topology of the ECM in situ will be key to understanding its role in tissue (patho)physiology. In order to see breakthroughs in the development of novel therapeutic interventions, it is crucial to understand the complex interactions and underlying feedback mechanisms that happen between the core matrisome, matrisome-associated factors and cells [168, 181]. Potential therapeutics for fibrotic diseases include enzymes that target ECM stiffness by degrading abnormal ECM, as well as antibodies that inhibit the activity of ECM cross-linking enzymes such as lysyl oxidases, and also the activity of MMPs and TGF- $\beta 1$ [73]. Furthermore, inhibitors for cellular receptors such as epidermal growth factor receptor, or drugs targeting the mechanotransduction machinery via integrins, are currently being tested as therapeutics in diseases such as lung cancer and fibrotic disorders [73). In addition, molecules and pathways involved in oxidative stress responses of the lung are being discussed as potential therapeutic targets [38]. There is also an increasing interest in using progenitor cells such as mesenchymal stem cells, which might contain regenerative potential, as enhancers for tissue repair [168]. As of now, however, lung transplantation remains the only valid therapy for end-stage lung diseases, but a shortage of donor lungs and the need for intense immunosuppression to obviate allograft rejection limit their clinical application. Therefore, bioartifical ECM scaffolds will become important elements of stem cell-based therapies in regenerative medicine. Future efforts will determine whether synthetic (3D-printed) scaffolds or decellularised ECM scaffolds of whole organs and their recellullarisation with autologous progenitor or stem cells may lead to fully functional and transplantable organs. Fabricating such lung surrogates is still far from reality, but basic research on ECM architecture and function, as well as on tissue engineering, is likely to yield new insights for translation into clinical use.

\section{References}

Hynes RO. The extracellular matrix: not just pretty fibrils. Science 2009; 326: 1216-1219.

Sorokin L. The impact of the extracellular matrix on inflammation. Nat Rev Immunol 2010; 10: 712-723.

Naba A, Hoersch S, Hynes RO. Towards definition of an ECM parts list: an advance on GO categories. Matrix Biol 2012; 31: 371-372.

4 Hynes RO, Naba A. Overview of the matrisome-an inventory of extracellular matrix constituents and functions. Cold Spring Harb Perspect Biol 2012; 4: a004903.

5 Naba A, Clauser KR, Hoersch S, et al. The matrisome: in silico definition and in vivo characterization by proteomics of normal and tumor extracellular matrices. Mol Cell Proteomics 2012; 11: M111.014647.

6 Naba A, Clauser KR, Lamar JM, et al. Extracellular matrix signatures of human mammary carcinoma identify novel metastasis promoters. Elife 2014; 3: e01308.

7 Naba A, Clauser KR, Hoersch S, et al. The matrisome: in silico definition and in vivo characterization by proteomics of normal and tumor extracellular matrices. Mol Cell Proteomics 2012; 11: M111 014647.

8 Rashid ST, Humphries JD, Byron A, et al. Proteomic analysis of extracellular matrix from the hepatic stellate cell line LX-2 identifies CYR61 and Wnt-5a as novel constituents of fibrotic liver. J Proteome Res 2012; 11: 4052-4064.

9 Zanivan S, Maione F, Hein MY, et al. SILAC-based proteomics of human primary endothelial cell morphogenesis unveils tumor angiogenic markers. Mol Cell Proteomics 2013; 12: 3599-3611.

10 Didangelos A, Yin X, Mandal K, et al. Proteomics characterization of extracellular space components in the human aorta. Mol Cell Proteomics 2010; 9: 2048-2062.

11 Barallobre-Barreiro J, Didangelos A, Schoendube FA, et al. Proteomics analysis of cardiac extracellular matrix remodeling in a porcine model of ischemia/reperfusion injury. Circulation 2012; 125: 789-802.

12 Schiller HB, Fernandez IE, Burgstaller G, et al. Time- and compartment-resolved proteome profiling of the extracellular niche in lung injury and repair. Mol Syst Biol 2015; 11: 819.

13 Booth AJ, Hadley R, Cornett AM, et al. Acellular normal and fibrotic human lung matrices as a culture system for in vitro investigation. Am J Respir Crit Care Med 2012; 186: 866-876.

14 Wisniewski JR, Hein MY, Cox J, et al. A "proteomic ruler" for protein copy number and concentration estimation without spike-in standards. Mol Cell Proteomics, 2014; 13: 3497-3506.

15 Suki B, Ito S, Stamenovic D, et al. Biomechanics of the lung parenchyma: critical roles of collagen and mechanical forces. J Appl Physiol (1985) 2005; 98: 1892-1899.

16 Suki B, Bates JH. Extracellular matrix mechanics in lung parenchymal diseases. Respir Physiol Neurobiol 2008; 163: 33-43.

17 Faffe DS, Zin WA. Lung parenchymal mechanics in health and disease. Physiol Rev 2009; 89: $759-775$.

18 Wagenseil JE, Mecham RP. New insights into elastic fiber assembly. Birth Defects Res C Embryo Today 2007; 81: $229-240$.

19 Sakai LY, Keene DR, Glanville RW, et al. Purification and partial characterization of fibrillin, a cysteine-rich structural component of connective tissue microfibrils. J Biol Chem 1991; 266: 14763-14770.

20 Wulf-Johansson H, Lock Johansson S, Schlosser A, et al. Localization of microfibrillar-associated protein 4 (MFAP4) in human tissues: clinical evaluation of serum MFAP4 and its association with various cardiovascular conditions. PLoS One 2013; 8: e82243.

21 Molleken C, Poschmann G, Bonella F, et al. MFAP4: a candidate biomarker for hepatic and pulmonary fibrosis? Sarcoidosis Vasc Diffuse Lung Dis 2016; 33: 41-50.

22 Frantz C, Stewart KM, Weaver VM. The extracellular matrix at a glance. J Cell Sci, 2010; 123(Pt 24): 4195-4200.

23 Bosman FT, Stamenkovic I. Functional structure and composition of the extracellular matrix. J Pathol 2003; 200: 423-428.

24 Humphrey JD, Dufresne ER, Schwartz MA. Mechanotransduction and extracellular matrix homeostasis. Nat Rev Mol Cell Biol 2014; 15: 802-812. 

415: $171-175$. the gC1q domain. J Biol Chem 2003; 278: 6160-6167.

27 Ruoslahti E, Pierschbacher MD. New perspectives in cell adhesion: RGD and integrins. Science 1987; 238: 491-497.

28 Dunsmore SE, Rannels DE. Extracellular matrix biology in the lung. Am J Physiol, 1996; 270: L3-L27.

29 Iozzo RV. Matrix proteoglycans: from molecular design to cellular function. Annu Rev Biochem 1998; 67: 609-652.

30 Kolb M, Margetts PJ, Sime PJ, et al. Proteoglycans decorin and biglycan differentially modulate TGF-beta-mediated fibrotic responses in the lung. Am J Physiol Lung Cell Mol Physiol 2001; 280: L1327-L1334.

31 Theocharis AD, Gialeli C, Bouris P, et al. Cell-matrix interactions: focus on proteoglycan-proteinase interplay and pharmacological targeting in cancer. FEBS J 2014; 281: 5023-5042.

32 Erler JT, Weaver VM. Three-dimensional context regulation of metastasis. Clin Exp Metastasis 2009; $26: 35-49$.

33 Cox TR, Bird D, Baker AM, et al. LOX-mediated collagen crosslinking is responsible for fibrosis-enhanced metastasis. Cancer Res 2013; 73: 1721-1732.

34 Xiao Q, Ge G. Lysyl oxidase, extracellular matrix remodeling and cancer metastasis. Cancer Microenviron 2012; 5: 261-273.

35 Iismaa SE, Mearns BM, Lorand L, et al. Transglutaminases and disease: lessons from genetically engineered mouse models and inherited disorders. Physiol Rev 2009; 89: 991-1023.

$36 \mathrm{Li}$ Y, Fessel G, Georgiadis M, et al. Advanced glycation end-products diminish tendon collagen fiber sliding. Matrix Biol 2013; 32: 169-177.

37 Jaisson S, Lorimier S, Ricard-Blum S, et al. Impact of carbamylation on type I collagen conformational structure and its ability to activate human polymorphonuclear neutrophils. Chem Biol 2006; 13: 149-159.

38 Watson WH, Ritzenthaler JD, Roman J. Lung extracellular matrix and redox regulation. Redox Biol 2016; 8: 305-315.

39 Daniil ZD, Papageorgiou E, Koutsokera A, et al. Serum levels of oxidative stress as a marker of disease severity in idiopathic pulmonary fibrosis. Pulm Pharmacol Ther 2008; 21: 26-31.

40 Montuschi P, Corradi M, Ciabattoni G, et al. Increased 8-isoprostane, a marker of oxidative stress, in exhaled condensate of asthma patients. Am J Respir Crit Care Med 1999; 160: 216-220.

41 Shelef MA, Bennin DA, Mosher DF, et al. Citrullination of fibronectin modulates synovial fibroblast behavior. Arthritis Res Ther 2012; 14: R240.

42 Seet BT, Dikic I, Zhou MM, et al. Reading protein modifications with interaction domains. Nat Rev Mol Cell Biol 2006; 7: 473-483.

43 Kristensen JH, Karsdal MA, Genovese F, et al. The role of extracellular matrix quality in pulmonary fibrosis. Respiration 2014; 88: 487-499.

44 Walsh G, Jefferis R. Post-translational modifications in the context of therapeutic proteins. Nat Biotechnol 2006; 24: $1241-1252$.

45 Leeming DJ, Bay-Jensen AC, Vassiliadis E, et al. Post-translational modifications of the extracellular matrix are key events in cancer progression: opportunities for biochemical marker development. Biomarkers 2011; 16: 193-205.

46 Hynes RO. Integrins: bidirectional, allosteric signaling machines. Cell 2002; 110: 673-687.

47 Schiller HB, Fassler R. Mechanosensitivity and compositional dynamics of cell-matrix adhesions. EMBO Rep 2013; 14: 509-519.

48 Geiger B, Spatz JP, Bershadsky AD. Environmental sensing through focal adhesions. Nat Rev Mol Cell Biol 2009; 10: 21-33.

49 Zaidel-Bar R, Itzkovitz S, Ma'ayan A, et al. Functional atlas of the integrin adhesome. Nat Cell Biol 2007; 9: $858-867$.

50 Schiller HB, Hermann MR, Polleux J, et al. $\beta_{1^{-}}$and $\alpha_{\mathrm{v}}$-class integrins cooperate to regulate myosin II during rigidity sensing of fibronectin-based microenvironments. Nat Cell Biol 2013; 15: 625-636.

51 Schiller HB, et al. Quantitative proteomics of the integrin adhesome show a myosin II-dependent recruitment of LIM domain proteins. EMBO Rep 2011; 12: 259-266.

52 Kuo JC, Han X, Hsiao CT, et al. Analysis of the myosin-II-responsive focal adhesion proteome reveals a role for beta-Pix in negative regulation of focal adhesion maturation. Nat Cell Biol 2011; 13: 383-393.

53 Horton ER, Byron A, Askari JA, et al. Definition of a consensus integrin adhesome and its dynamics during adhesion complex assembly and disassembly. Nat Cell Biol 2015; 17: 1577-1587.

54 Geiger T, Zaidel-Bar R. Opening the floodgates: proteomics and the integrin adhesome. Curr Opin Cell Biol 2012; 24: 562-568.

55 Humphries JD, Byron A, Bass MD, et al. Proteomic analysis of integrin-associated complexes identifies RCC2 as a dual regulator of Rac1 and Arf6. Sci Signal 2009; 2: ra51.

56 Connelly JT, Gautrot JE, Trappmann B, et al. Actin and serum response factor transduce physical cues from the microenvironment to regulate epidermal stem cell fate decisions. Nature Cell Biol 2010; 12: 711-718.

57 Zhou Y, Huang X, Hecker L, et al. Inhibition of mechanosensitive signaling in myofibroblasts ameliorates experimental pulmonary fibrosis. J Clin Invest 2013; 123: 1096-1108.

58 Lu P, Weaver VM, Werb Z. The extracellular matrix: A dynamic niche in cancer progression. J Cell Biol 2012; 196: 395-406.

59 Levental KR, Yu H, Kass L, et al. Matrix crosslinking forces tumor progression by enhancing integrin signaling. Cell 2009; 139: 891-906.

60 Huang X, Gai Y, Yang N, et al. Relaxin regulates myofibroblast contractility and protects against lung fibrosis Am J Pathol 2011; 179: 2751-2765.

61 Kai F, Laklai H, Weaver VM. Force matters: biomechanical regulation of cell invasion and migration in disease. Trends Cell Biol 2016; 26: 486-497.

62 Engler A, Bacakova L, Newman C, et al. Substrate compliance versus ligand density in cell on gel responses. Biophys J, 2004; 86: 617-628. 
63 Lo CM, et al. Cell movement is guided by the rigidity of the substrate. Biophys J 2000; 79: 144-152.

64 Vincent LG, Choi YS, Alonso-Latorre B, et al. Mesenchymal stem cell durotaxis depends on substrate stiffness gradient strength. Biotechnol J 2013; 8: 472-484.

65 Lee S, Hong J, Lee J. Cell motility regulation on a stepped micro pillar array device (SMPAD) with a discrete stiffness gradient. Soft Matter 2016; 12: 2325-2333.

66 Fu J, Wang YK, Yang MT, et al. Mechanical regulation of cell function with geometrically modulated elastomeric substrates. Nat Methods 2010; 7: 733-736.

67 Goffin JM, Pittet P, Csucs G, et al. Focal adhesion size controls tension-dependent recruitment of alpha-smooth muscle actin to stress fibers. J Cell Biol 2006; 172: 259-268.

68 Liu F, Mih JD, Shea BS, et al. Feedback amplification of fibrosis through matrix stiffening and COX-2 suppression. J Cell Biol 2010; 190: 693-706.

69 Arnaout MA, Goodman SL, Xiong JP. Structure and mechanics of integrin-based cell adhesion. Curr Opin Cell Biol 2007; 19: 495-507.

$70 \quad$ Patel NR, Bole M, Chen C, et al. Cell elasticity determines macrophage function. PLoS One 2012; 7: e41024.

71 Krishnan R, Klumpers DD, Park CY, et al. Substrate stiffening promotes endothelial monolayer disruption through enhanced physical forces. Am J Physiol Cell Physiol 2011; 300: C146-C154.

72 Taipale J, Keski-Oja J. Growth factors in the extracellular matrix. Faseb J 1997; 11: 51-59.

73 Bonnans C, Chou J, Werb Z. Remodelling the extracellular matrix in development and disease. Nat Rev Mol Cell Biol 2014; 15: 786-801.

74 Overall CM. Molecular determinants of metalloproteinase substrate specificity: matrix metalloproteinase substrate binding domains, modules, and exosites. Mol Biotechnol 2002; 22: 51-86.

75 Sand JM, Larsen L, Hogaboam C, et al. MMP mediated degradation of type IV collagen alpha 1 and alpha 3 chains reflects basement membrane remodeling in experimental and clinical fibrosis-validation of two novel biomarker assays. PLoS One 2013; 8: e84934.

Willenbrock F, Crabbe T, Slocombe PM, et al. The activity of the tissue inhibitors of metalloproteinases is regulated by C-terminal domain interactions: a kinetic analysis of the inhibition of gelatinase A. Biochemistry 1993; 32: 4330-4337.

77 van Goor H, Melenhorst WBWH, Turner AJ, et al. Adamalysins in biology and disease. J Pathol 2009; 219: 277-286.

78 Byrne AJ, Mathie SA, Gregory LG, et al. Pulmonary macrophages: key players in the innate defence of the airways. Thorax 2015; 70: 1189-1196.

79 Pardo A, Cabrera S, Maldonado M, et al. Role of matrix metalloproteinases in the pathogenesis of idiopathic pulmonary fibrosis. Respir Res 2016; 17: 23.

80 Zuo F, Kaminski N, Eugui E, et al. Gene expression analysis reveals matrilysin as a key regulator of pulmonary fibrosis in mice and humans. Proc Natl Acad Sci USA 2002; 99: 6292-6297.

81 Lu P, Takai K, Weaver VM, et al. Extracellular matrix degradation and remodeling in development and disease. Cold Spring Harb Perspect Biol 2011; 3(12).

82 Richeldi L, du Bois RM, Raghu G, et al. Efficacy and safety of nintedanib in idiopathic pulmonary fibrosis. $N$ Engl J Med 2014; 370: 2071-2082.

83 King TE Jr, Bradford WZ, Castro-Bernardini S, et al. A phase 3 trial of pirfenidone in patients with idiopathic pulmonary fibrosis. N Engl J Med 2014; 370: 2083-2092.

84 Noble PW, Barkauskas CE, Jiang D. Pulmonary fibrosis: patterns and perpetrators. J Clin Invest 2012; 122: 2756-2762.

85 Wolters PJ, Collard HR, Jones KD. Pathogenesis of idiopathic pulmonary fibrosis. Annu Rev Pathol 2014; 9: 157-179.

86 Fernandez IE, Eickelberg O. New cellular and molecular mechanisms of lung injury and fibrosis in idiopathic pulmonary fibrosis. Lancet 2012; 380: 680-688.

87 Hashimoto N, Jin H, Liu T, et al. Bone marrow-derived progenitor cells in pulmonary fibrosis. J Clin Invest 2004; 113: 243-252.

88 Quan TE, Cowper S, Wu SP, et al. Circulating fibrocytes: collagen-secreting cells of the peripheral blood. Int $J$ Biochem Cell Biol 2004; 36: 598-606.

89 Bellini A, Mattoli S. The role of the fibrocyte, a bone marrow-derived mesenchymal progenitor, in reactive and reparative fibroses. Lab Invest 2007; 87: 858-870.

90 Willis BC, Liebler JM, Luby-Phelps K, et al. Induction of epithelial-mesenchymal transition in alveolar epithelial cells by transforming growth factor-beta1: potential role in idiopathic pulmonary fibrosis. Am J Pathol 2005; 166: $1321-1332$.

91 Tsukui T, Ueha S, Shichino S, et al. Intratracheal cell transfer demonstrates the profibrotic potential of resident fibroblasts in pulmonary fibrosis. Am J Pathol 2015; 185: 2939-2948.

92 Hung C, Linn G, Chow YH, et al. Role of lung pericytes and resident fibroblasts in the pathogenesis of pulmonary fibrosis. Am J Respir Crit Care Med 2013; 188: 820-830.

93 Rock JR, Barkauskas CE, Cronce MJ, et al. Multiple stromal populations contribute to pulmonary fibrosis without evidence for epithelial to mesenchymal transition. Proc Natl Acad Sci USA 2011; 108: E1475-E1483.

94 Parker MW, Rossi D, Peterson M, et al. Fibrotic extracellular matrix activates a profibrotic positive feedback loop. J Clin Invest 2014 124:1622-1635.

95 Ott HC, Clippinger B, Conrad C, et al. Regeneration and orthotopic transplantation of a bioartificial lung. Nat Med 2010; 16: 927-933.

96 Song JJ, Kim SS, Liu Z, et al. Enhanced in vivo function of bioartificial lungs in rats. Ann Thorac Surg 2011; 92: 998-1005.

97 Wagner DE, Bonvillain RW, Jensen T, et al. Can stem cells be used to generate new lungs? Ex vivo lung bioengineering with decellularized whole lung scaffolds. Respirology 2013; 18: 895-911.

98 Wagner DE, Bonenfant NR, Parsons CS, et al. Comparative decellularization and recellularization of normal versus emphysematous human lungs. Biomaterials 2014; 35: 3281-3297.

99 Malanchi I, Santamaria-Martínez A, Susanto E, et al. Interactions between cancer stem cells and their niche govern metastatic colonization. Nature 2012; 481: 85-89. 
Baghy K, Iozzo RV, Kovalszky I. Decorin-TGF $\beta$ axis in hepatic fibrosis and cirrhosis. J Histochem Cytochem 2012; 60: 262-268.

Marastoni S, Andreuzzi E, Paulitti A, et al. EMILIN2 down-modulates the Wnt signalling pathway and suppresses breast cancer cell growth and migration. J Pathol 2014; 232: 391-404.

Calderwood CJ, Jones MG, Hoile L, et al. Secreted lysyl oxidase is elevated in the bronchoalveolar lavage fluid of patients with idiopathic pulmonary fibrosis. Thorax 2012; 67: A111.

Olsen KC, Sapinoro RE, Kottmann RM, et al. Transglutaminase 2 and its role in pulmonary fibrosis. Am J Respir Crit Care Med 2011; 184: 699-707.

Henderson NC, Arnold TD, Katamura Y, et al. Targeting of $\alpha_{\mathrm{v}}$ integrin identifies a core molecular pathway that regulates fibrosis in several organs. Nat Med 2013; 19: 1617-1624.

enkins RG, Su X, Su G, et al. Ligation of protease-activated receptor 1 enhances alphavbeta6 integrin-dependent TGF- $\beta$ activation and promotes acute lung injury. J Clin Invest 2006; 116: 1606-1614.

Decaris ML, Gatmaitan M, FlorCruz S, et al. Proteomic analysis of altered extracellular matrix turnover in bleomycin-induced pulmonary fibrosis. Mol Cell Proteomics 2014; 13: 1741-1752.

Gooptu B, Ekeowa UI, Lomas DA. Mechanis
cellular insights. Eur Respir J 2009; 34: 475-488.

Snider GL, Lucey EC, Stone PJ. Animal models of emphysema. Am Rev Respir Dis 1986; 133: 149-169.

Suki B, Sato S, Parameswaran H, et al. Emphysema and mechanical stress-induced lung remodeling. Physiology (Bethesda) 2013; 28: 404-413.

Ito S, Ingenito EP, Brewer KK, et al. Mechanics, nonlinearity, and failure strength of lung tissue in a mouse model of emphysema: possible role of collagen remodeling. J Appl Physiol (1985) 2005; 98: 503-511.

Finlay GA, O'Donnell MD, O'Connor CM, et al. Elastin and collagen remodeling in emphysema. A scanning electron microscopy study. Am J Pathol 1996; 149: 1405-1415.

Pacini A, Gardi C, Corradeschi $\mathrm{F}$, et al. In vivo stimulation of lung collagen synthesis by collagen derived peptides. Res Commun Chem Pathol Pharmacol 1990; 68: 89-101.

Vlahovic G, Russell ML, Mercer RR, et al. Cellular and connective tissue changes in alveolar septal walls in emphysema. Am J Respir Crit Care Med 1999; 160: 2086-2092. 40: $1362-1373$

Roman J, Ritzenthaler JD, Gil-Acosta A, et al. Nicotine and fibronectin expression in lung fibroblasts: implications for tobacco-related lung tissue remodeling. FASEB J 2004; 18: 1436-1438.

Chen L, Ge Q, Tjin G, et al. Effects of cigarette smoke extract on human airway smooth muscle cells in COPD. Eur Respir J 2014; 44: 634-646.

Ramnath NW, van de Luijtgaarden KM, van der Pluijm I, et al. Extracellular matrix defects in aneurysmal Fibulin-4 mice predispose to lung emphysema. PLoS One 2014; 9: e106054.

Papaporfyriou A, Loukides S, Kostikas K, et al. Increased levels of osteopontin in sputum supernatant in patients with COPD. Chest 2014; 146: 951-958.

Pardo A, Gibson K, Cisneros J, et al. Up-regulation and profibrotic role of osteopontin in human idiopathic pulmonary fibrosis. PLoS Med 2005; 2: e251.

Hoonhorst SJ, Lo Tam Loi AT, Hartman JE, et al. Advanced glycation end products in the skin are enhanced in COPD. Metabolism 2014; 63: 1149-1156.

Sand JM, Knox AJ, Lange P, et al. Accelerated extracellular matrix turnover during exacerbations of COPD Respir Res 2015; 16: 69.

Jonigk D, Golpon H, Bockmeyer CL, et al. Plexiform lesions in pulmonary arterial hypertension composition, architecture, and microenvironment. Am J Pathol 2011; 179: 167-179.

Stenmark KR, Meyrick B, Galie N, et al. Animal models of pulmonary arterial hypertension: the hope for etiological discovery and pharmacological cure. Am J Physiol Lung Cell Mol Physiol 2009; 297: L1013-L1032.

Poiani GJ, Tozzi CA, Yohn SE, et al. Collagen and elastin metabolism in hypertensive pulmonary arteries of rats. Circ Res 1990; 66: 968-978.

Jones PL, Cowan KN, Rabinovitch M. Tenascin-C, proliferation and subendothelial fibronectin in progressive pulmonary vascular disease. Am J Pathol 1997; 150: 1349-1360.

Karnik SK, Brooke BS, Bayes-Genis A, et al. A critical role for elastin signaling in vascular morphogenesis and disease. Development 2003; 130: 411-423.

Papakonstantinou E, Kouri FM, Karakiulakis G, et al. Increased hyaluronic acid content in idiopathic pulmonary arterial hypertension. Eur Respir J 2008; 32: 1504-1512.

Kalay N, Elcik D, Canatan H, et al. Elevated plasma hyaluronan levels in pulmonary hypertension. Tohoku J Exp Med 2013; 230: 7-11.

Lauer ME, Aytekin M, Comhair SA, et al. Modification of hyaluronan by heavy chains of inter-alpha-inhibitor in idiopathic pulmonary arterial hypertension. J Biol Chem 2014; 289: 6791-6798.

Lepetit H, Eddahibi S, Fadel E, et al. Smooth muscle cell matrix metalloproteinases in idiopathic pulmonary arterial hypertension. Eur Respir J 2005; 25: 834-842.

Coen M, Gabbiani G, Bochaton-Piallat ML. Myofibroblast-mediated adventitial remodeling: an underestimated player in arterial pathology. Arterioscler Thromb Vasc Biol 2011; 31: 2391-2396.

Nave AH, Mižíková I, Niess G, et al. Lysyl oxidases play a causal role in vascular remodeling in clinical and experimental pulmonary arterial hypertension. Arterioscler Thromb Vasc Biol 2014; 34: 1446-1458.

Scarritt ME, Bonvillain RW, Burkett BJ, et al. Hypertensive rat lungs retain hallmarks of vascular disease upon decellularization but support the growth of mesenchymal stem cells. Tissue Eng Part A 2014; 20: 1426-1443.

Mauad T, Silva LF, Santos MA, et al. Abnormal alveolar attachments with decreased elastic fiber content in distal lung in fatal asthma. Am J Respir Crit Care Med 2004; 170: 857-862.

Roche WR, Beasley R, Williams JH, et al. Subepithelial fibrosis in the bronchi of asthmatics. Lancet 1989; 1: $520-524$.

Araujo BB, Dolhnikoff M, Silva LF, et al. Extracellular matrix components and regulators in the airway smooth muscle in asthma. Eur Respir J 2008; 32: 61-69. 
Weitoft M, Andersson C, Andersson-Sjöland A, et al. Controlled and uncontrolled asthma display distinct alveolar tissue matrix compositions. Respir Res 2014; 15: 67.

Nihlberg K, Andersson-Sjöland A, Tufvesson E, et al. Altered matrix production in the distal airways of individuals with asthma. Thorax 2010; 65: 670-676.

Dekkers BG, Schaafsma D, Nelemans A, et al. Extracellular matrix proteins differentially regulate airway smooth muscle phenotype and function. Am J Physiol Lung Cell Mol Physiol 2007; 292: L1405-L1413.

Johnson PR, Burgess JK, Underwood PA, et al. Extracellular matrix proteins modulate asthmatic airway smooth muscle cell proliferation via an autocrine mechanism. J Allergy Clin Immunol 2004; 113: 690-696.

Hanahan D, Weinberg RA. Hallmarks of cancer: the next generation. Cell 2011; 144: 646-674.

Pickup MW, Mouw JK, Weaver VM. The extracellular matrix modulates the hallmarks of cancer. EMBO Rep 2014; 15: 1243-1253.

Sethi T, Rintoul RC, Moore SM, et al. Extracellular matrix proteins protect small cell lung cancer cells against apoptosis: a mechanism for small cell lung cancer growth and drug resistance in vivo. Nat Med 1999; 5: 662-668. Sivridis E, Giatromanolaki A, Koukourakis MI. "Stromatogenesis" and tumor progression. Int J Surg Pathol 2004; 12: $1-9$.

Chen Y, Terajima M, Yang Y, et al. Lysyl hydroxylase 2 induces a collagen cross-link switch in tumor stroma. J Clin Invest 2015; 125: 1147-1162.

Barker HE, Cox TR, Erler JT. The rationale for targeting the LOX family in cancer. Nat Rev Cancer 2012; 12 $540-552$.

Mouw JK, Yui Y, Damiano L, et al. Tissue mechanics modulate microRNA-dependent PTEN expression to regulate malignant progression. 2014; 20: 360-367.

8: $241-254$

Whipple CA. Tumor talk: understanding the conversation between the tumor and its microenvironment. Cancer Cell Microenviron 2015; 2: e773.

Vancheri C. Idiopathic pulmonary fibrosis and cancer: do they really look similar? BMC Med 2015; $13: 220$.

Vancheri C, Failla M, Crimi N, et al. Idiopathic pulmonary fibrosis: a disease with similarities and links to cancer biology. Eur Respir J 2010; 35: 496-504.

Vancheri C. Idiopathic pulmonary fibrosis: an altered fibroblast proliferation linked to cancer biology. Proc Am Thorac Soc 2012; 9: 153-157.

Antoniou KM, Tomassetti S, Tsitoura E, et al. Idiopathic pulmonary fibrosis and lung cancer: a clinical and pathogenesis update. Curr Opin Pulm Med 2015; 21: 626-633.

Vancheri C. Common pathways in idiopathic pulmonary fibrosis and cancer. Eur Respir Rev 2013; 22: $265-272$. Burgstaller G, Oehrle B, Koch I, et al. Multiplex profiling of cellular invasion in 3D cell culture models. PLoS One 2013; 8: e63121.

Marques-Garcia F, Marcos-Vadillo E. Review of mouse models applied to the study of asthma. Methods Mol Biol 2016; 1434: 213-222.

Nogueira-Ferreira R, Vitorino R, Ferreira R, et al. Exploring the monocrotaline animal model for the study of pulmonary arterial hypertension: A network approach. Pulm Pharmacol Ther 2015; 35: 8-16.

Ryan JJ, Marsboom G, Archer SL. Rodent models of group 1 pulmonary hypertension. Handb Exp Pharmacol 2013; 218: 105-149.

Gazdar AF, Hirsch FR, Minna JD. From mice to men and back: an assessment of preclinical model systems for the study of lung cancers. J Thorac Oncol 2016; 11: 287-299.

Fricker M, Deane A, Hansbro PM. Animal models of chronic obstructive pulmonary disease. Expert Opin Drug Discov 2014; 9: 629-645.

Mizikova I, Morty RE. The extracellular matrix in bronchopulmonary dysplasia: target and source. Front Med (Lausanne) 2015; 2: 91.

Moore B, Lawson WE, Oury TD, et al. Animal models of fibrotic lung disease. Am J Respir Cell Mol Biol 2013; 49: 167-179.

Cukierman E, Pankov R, Yamada KM. Cell interactions with three-dimensional matrices. Curr Opin Cell Biol 2002; 14: 633-639.

Baker BM, Chen CS. Deconstructing the third dimension: how 3D culture microenvironments alter cellular cues. J Cell Sci, 2012; 125: 3015-3024.

Abbott A. Cell culture: biology's new dimension. Nature 2003; 424: 870-872.

Thannickal VJ, Henke CA, Horowitz JC, et al. Matrix biology of idiopathic pulmonary fibrosis: a workshop of the National Heart, Lung, and Blood Institute. Am J Pathol 2014; 184: 1643-1651.

Hinz B. Mechanical aspects of lung fibrosis: a spotlight on the myofibroblast. Proc Am Thorac Soc 2012; 9: 137-147.

White ES. Lung extracellular matrix and fibroblast function. Ann Am Thorac Soc, 2015; 12 Suppl. 1: S30-S33.

Saums MK, Wang W, Han B, et al. Mechanically and chemically tunable cell culture system for studying the myofibroblast phenotype. Langmuir 2014; 30: 5481-5487.

Silver FH, Pins G. Cell growth on collagen: a review of tissue engineering using scaffolds containing extracellular matrix. J Long Term Eff Med Implants 1992; 2: 67-80.

Hafemann B, Ensslen S, Erdmann C, et al. Use of a collagen/elastin-membrane for the tissue engineering of dermis. Burns 1999; 25: 373-384.

Hayen W, Goebeler M, Kumar S, et al. Hyaluronan stimulates tumor cell migration by modulating the fibrin fiber architecture. J Cell Sci 1999; 112: 2241-2251.

Rodin S, Antonsson L, Niaudet C, et al. Clonal culturing of human embryonic stem cells on laminin-521/ E-cadherin matrix in defined and xeno-free environment. Nat Commun 2014; 5: 3195. proteolytically mediated cell migration. Biophys J 2005; 89: 1374-1388. caveolin-1 favors tumor invasion and metastasis. Cell 2011; 146: 148-163. 
176 Uhl FE, Vierkotten S, Wagner DE, et al. Preclinical validation and imaging of Wnt-induced repair in human 3D lung tissue cultures. Eur Respir J 2015; 46: 1150-1166.

177 Burgstaller G, Vierkotten S, Lindner M, et al. Multidimensional immunolabeling and 4D time-lapse imaging of vital ex vivo lung tissue. Am J Physiol Lung Cell Mol Physiol 2015; 309: L323-L332.

178 Donovan C, Bailey SR, Tran J, et al. Rosiglitazone elicits in vitro relaxation in airways and precision cut lung slices from a mouse model of chronic allergic airways disease. Am J Physiol Lung Cell Mol Physiol 2015; 309: L1219-L1228.

179 Goldstein TA, Smith BD, Zeltsman D, et al. Introducing a 3-dimensionally printed, tissue-engineered graft for airway reconstruction: a pilot study. Otolaryngol Head Neck Surg 2015; 15: 1001-1006.

180 Psaila B, Lyden D. The metastatic niche: adapting the foreign soil. Nat Rev Cancer 2009; 9: 285-293.

181 Burgess JK, Mauad T, Tjin G, et al. The extracellular matrix - the under-recognized element in lung disease? J Pathol 2016; 240: 397-409. 